\title{
Safety and efficacy of repeat long-term incobotulinumtoxinA treatment for lower limb or combined upper/lower limb spasticity in children with cerebral palsy
}

\author{
Petr Kaňovskýa,*, Florian Heinen ${ }^{\mathrm{b}}$, A. Sebastian Schroeder ${ }^{\mathrm{b}}$, Henry G. Chambers ${ }^{\mathrm{c}}$, \\ Edward Dabrowski $^{\mathrm{d}}$, Thorin L. Geister ${ }^{\mathrm{e}}$, Angelika Hanschmann ${ }^{\mathrm{e}}$, Francisco J. Martinez-Torres ${ }^{\mathrm{f}}$, \\ Irena Pulte ${ }^{\mathrm{e}}$, Marta Banach ${ }^{\mathrm{g}}$ and Deborah Gaebler-Spira ${ }^{\mathrm{h}}$ \\ ${ }^{a}$ Faculty of Medicine and Dentistry and University Hospital, Palacký University Olomouc, Olomouc, Czech \\ Republic \\ ${ }^{\mathrm{b}}$ Department of Pediatric Neurology \& Developmental Medicine and LMU Center for Children with Medical \\ Complexity, Dr. von Hauner Children's Hospital, Ludwig-Maximilians-University Munich, Munich, Germany \\ ${ }^{\mathrm{c}}$ Rady Children's Hospital, San Diego, CA, USA \\ ${ }^{\mathrm{d}}$ Beaumont Pediatric Physical Medicine \& Rehabilitation - Royal Oak, Royal Oak, MI, USA \\ ${ }^{\mathrm{e}}$ Merz Pharmaceuticals GmbH, Frankfurt am Main, Germany \\ ${ }^{\mathrm{f}}$ Formerly of Merz North America, Raleigh, NC, USA

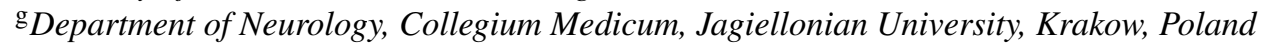 \\ ${ }^{\mathrm{h}}$ Shirley Ryan AbilityLab, Chicago, IL, USA
}

Received 13 April 2021

Accepted 28 September 2021

\begin{abstract}
.
PURPOSE: The open-label phase 3 "Treatment with IncobotulinumtoxinA in Movement Open-Label" (TIMO) study investigated longer-term safety and efficacy of incobotulinumtoxinA in children/adolescents with cerebral palsy (CP).

METHODS: Patients on standard treatment, with unilateral or bilateral lower limb (LL) or combined upper limb (UL)/LL spasticity received four incobotulinumtoxinA injection cycles (16 or 20 Units $/ \mathrm{kg}$ bodyweight total [maximum 400 or 500 Units] per cycle depending on ambulatory status/clinical pattern treated), each followed by 12-16 weeks' observation. Treatment for pes equinus was mandatory; flexed knee or adducted thigh were options for unilateral treatment and/or ULs for unilateral/bilateral treatment. The primary endpoint was safety; changes in Ashworth Scale and Gross Motor Function Measure-66 scores, and Global Impression of Change Scale scores at week 4 of each injection cycle were also evaluated.

RESULTS: IncobotulinumtoxinA ( $\leq 500$ Units for $\leq 98$ weeks) was safe, well-tolerated, and effective across all endpoints for multipattern treatment of LL and combined LL/UL spasticity in ambulant/nonambulant children/adolescents with CP. Treatment effects increased with each injection cycle. No new/unexpected safety concerns were identified.

CONCLUSION: IncobotulinumtoxinA showed a good safety and tolerability profile, with efficacy over multiple clinical presentations. As an adjunct treatment, it offers an effective, individualized treatment option for pediatric CP-related spasticity.
\end{abstract}

Keywords: Botulinum toxins, Type A, muscle spasticity, movement disorders, paediatric, cerebral palsy

\footnotetext{
*Corresponding author: Petr Kaňovský, Faculty of Medicine and Dentistry and University Hospital, Palacký University
}

Olomouc, Olomouc, Czech Republic. Tel.: +420588 443400; E-mail: petr.kanovsky@fnol.cz. 


\section{Introduction}

Spasticity is the most frequent symptom of cerebral palsy (CP) in children [1], presenting in 70-90\% of those affected [2,3]. It has a major impact, limiting the range of passive and active joint motion and hindering the development of motor functions, rehabilitation, and patient care, as well as causing pain and reducing self-esteem and quality of life [1]. Spasticity can affect the entire body; children with bilateral involvement are usually more affected in the lower limbs (LLs), whereas those with unilateral spasticity are usually more affected in the upper limbs (ULs) [4]. In children with LL spasticity, the most prominent and most frequently observed presentation is pes equinus [5], which affects as many as $83 \%$ of children with bilateral spastic CP [6]; however, knee flexion and hip flexion or adduction are also common [7].

The management of $\mathrm{CP}$ is multidisciplinary, with impaired motor function being addressed using occupational and physical therapy and orthopedic interventions [8]. Botulinum toxin can be used to reduce hypertonia associated with spasticity, thereby increasing the range of passive and active motion, facilitating posture correction, and reducing discomfort and pain (the last two can also be problematic during rehabilitation) [1]. Botulinum neurotoxin type $\mathrm{A}$ (BoNT-A) injections are established, effective, and well-tolerated treatment options that should be used as part of a comprehensive, multimodal, interdisciplinary approach that includes other therapies to potentially give children with $\mathrm{CP}$ clinically meaningful benefits [9-13]. Clinically relevant treatment goals vary according to the patient's needs and are related to Gross Motor Function Classification System (GMFCS) level [10].

BoNT-A is recommended for use in children/ adolescents with a range of presentations of CP [9, 10], most commonly those with gait dysfunction such as pes equinus [10]. Indeed, heterogeneous groups of muscles and joints (clinical patterns) that can involve the LLs or ULs often need to be treated, depending on the patient's needs. The BoNT-A products currently available in the European Union and North America are onabotulinumtoxinA $[14,15]$, abobotulinumtoxinA [16, 17] and incobotulinumtoxinA [18, 19]. Specific indications for these BoNT-As vary by region and product. Studies confirming the efficacy of onabotulinumtoxinA and abobotulinumtoxinA were conducted in children/adolescents with $\mathrm{CP}$ and either UL or LL spasticity [11, 20-22]. However, studies that report the effects of BoNT-A treatment in chil- dren/adolescents with CP and multipattern spasticity affecting more than one LL and/or UL are limited. It is therefore important that the effects of BoNT-A are evaluated in a wide range of ambulant (GMFCS levels I-III) and nonambulant (GMFCS levels IV-V) children with CP and LL and/or UL spasticity involving different clinical patterns.

IncobotulinumtoxinA is a highly purified formulation of BoNT-A $(150 \mathrm{kD})$ that is free from complexing proteins; reduction of the overall bacterial protein load may be associated with lower immunogenicity [23]. A large, international phase 3 pediatric study program was initiated to investigate the efficacy and safety of incobotulinumtoxinA for the treatment of CP-related spasticity. Results of two doubleblind, randomized, parallel-group studies (Treatment with IncobotulinumtoxinA in Movement [TIM], NCT01893411; incobotulinumtoXinA in aRm treatment in cerebral pAlsy [XARA], NCT02002884) from this program showed incobotulinumtoxinA is effective and safe for the treatment of LL and/or UL spasticity in children and adolescents with CP and provided evidence for its efficacy in multipattern treatment, reflecting the real-world clinical needs of children with CP [24, 25].

The 'Treatment with IncobotulinumtoxinA in Movement Open-Label' (TIMO) study investigated the longer-term safety and efficacy of incobotulinumtoxinA in children/adolescents with LL or combined LL and UL spasticity due to CP (GMFCS expanded and revised [GMFCS-E\&R] levels I-V). A subset of patients with LL spasticity only who were included in TIMO had previously been treated with two injection cycles of incobotulinumtoxinA in the TIM study. Treatment was standardized for each clinical pattern, but individualized multipattern injections were allowed in both clinical trials, together with some flexibility in dosing based on the underlying spasticity.

\section{Methods}

The TIMO study was an open-label, noncontrolled, multicenter, phase 3 study conducted in 30 sites across 12 countries. Patients with uni- or bilateral LL spasticity due to $\mathrm{CP}$ who had completed the doubleblind, randomized, parallel-group phase 3 TIM study, or newly recruited patients with $\mathrm{CP}$ and uni- or bilateral LL or with combined UL and LL spasticity, were enrolled. Standardized treatment plans with predefined dose ranges and injection-site numbers for each muscle ensured homogeneity for each muscle 
group to enable a comparison of treatment efficacy.

Patients who were eligible to enter TIMO from TIM were treated for 74-98 weeks, with up to six injection cycles in total across both studies (Fig. 1a). Newly recruited patients had a 48- to 64-week treatment period with four injection cycles.

\subsection{Standard protocol approvals, registrations, and patient consent}

This study was conducted in accordance with good clinical practice and the ethical principles of the Declaration of Helsinki and registered on clinicaltrials.gov (NCT01905683). The study protocol, informed consent forms, and other appropriate studyrelated documents were reviewed and approved by the local independent ethics committees and institutional review boards. Parent(s)/guardian(s) of all patients provided written informed consent, and patients provided assent (if applicable).

\subsection{Participants}

Eligible patients were aged 2-17 years and either had completed TIM or were newly recruited with unior bilateral spasticity due to CP.

Patients who had completed TIM [24] were eligible for entry into TIMO if they 1) had uni- or bilateral LL pes equinus (unilateral pes equinus required subjects to have ipsilateral flexed knee or adducted thigh), 2) were treated with any TIM study dosage of incobotulinumtoxinA for two injection cycles of 12-16 weeks, and 3) had an Ashworth Scale (AS) score $\geq 2$ in the plantar flexors (at least unilaterally); patients with an AS score of 1 could be enrolled if the investigator determined a clinical need for reinjection. Recruitment from TIM was competitive and not guaranteed.

Although all patients were required to have pes equinus in at least one limb, newly recruited patients could have a wider range of clinical patterns than those enrolled from TIM (Fig. 1b): LL spasticity only (unilateral or bilateral pes equinus; those with unilateral pes equinus could also be treated for ipsilateral flexed knee or adducted thigh) or combined unilateral UL and uni- or bilateral LL spasticity (i.e., unilateral pes equinus plus ipsilateral clinical pattern flexed knee or adducted thigh plus flexed elbow, flexed wrist, clenched fist, thumb-in-palm and/or pronated forearm, or bilateral pes equinus plus the aforementioned options for UL clinical patterns). Newly recruited patients had an AS score $\geq 2$ in at least one plantar flexor and could be treatment naïve (no BoNT-A treatment within the last 12 months before study injections for treatment of LL spasticity) or pre-treated (no BoNT-A treatment within the last 16 weeks prior to first study treatment for any indication).

Key exclusion criteria of the TIM and TIMO studies included fixed contracture or predominant forms of muscle hypertonia other than spasticity (e.g., dystonia) in the target limb(s), surgery for pes equinus in the target limbs within 12 months prior to screening or planned within the study period, hip flexion requiring BoNT-A injection, limitation of hip abduction to $<40^{\circ}$ or a pre-diagnosed migrational percentage $>30^{\circ}$, a severe neurological diagnosis and comorbidity outside the spectrum of $\mathrm{CP}$, and pure dyskinetic $\mathrm{CP}$ or mixed $\mathrm{CP}$ with predominantly dyskinetic movements. Treatment with intrathecal baclofen, oral anticoagulants, or drugs acting as peripheral muscle relaxants was not allowed.

\subsection{Treatment}

Eligible patients received four incobotulinumtoxinA injection cycles, each followed by 12-16 weeks of observation (Fig. 1a). All patients were treated for pes equinus at least unilaterally throughout the study, but further clinical patterns could also be chosen as explained below.

Patients who entered the study from TIM received four uni- or bilateral LL intramuscular injection cycles of incobotulinumtoxinA $(8 \mathrm{U} / \mathrm{kg}$ per pattern, with a total dose per cycle of $16 \mathrm{U} / \mathrm{kg}$ bodyweight [BW]; maximum $400 \mathrm{U}$ ); this was the highest dose regimen utilized in TIM. Patients were treated according to the same clinical patterns selected in TIM based on individual clinical need, but this dose level was irrespective of their previous treatment regimen in TIM (Fig. 1b). At least two of the five plantar flexor muscles contributing to pes equinus (gastrocnemius, soleus, tibialis posterior, flexor digitorum longus, and flexor hallucis longus) were treated.

Newly recruited patients received intramuscular injections of incobotulinumtoxinA according to four standardized treatment combinations (Fig. 1b) chosen by the investigator at the first injection, depending on the patient's clinical need. The total body dose of 16-20 U/kg BW (maximum 400-500 U) for each injection cycle was dependent on the patient's ambulatory status: nonambulatory patients (GMFCS-E\&R levels IV-V) received a total body dose restricted to $16 \mathrm{U} / \mathrm{kg} \mathrm{BW}$ (maximum total dose of $400 \mathrm{U}$ ) for safety reasons. LL clinical patterns (pes equinus, flexed knee, and adducted thigh) could be treated 
(a)

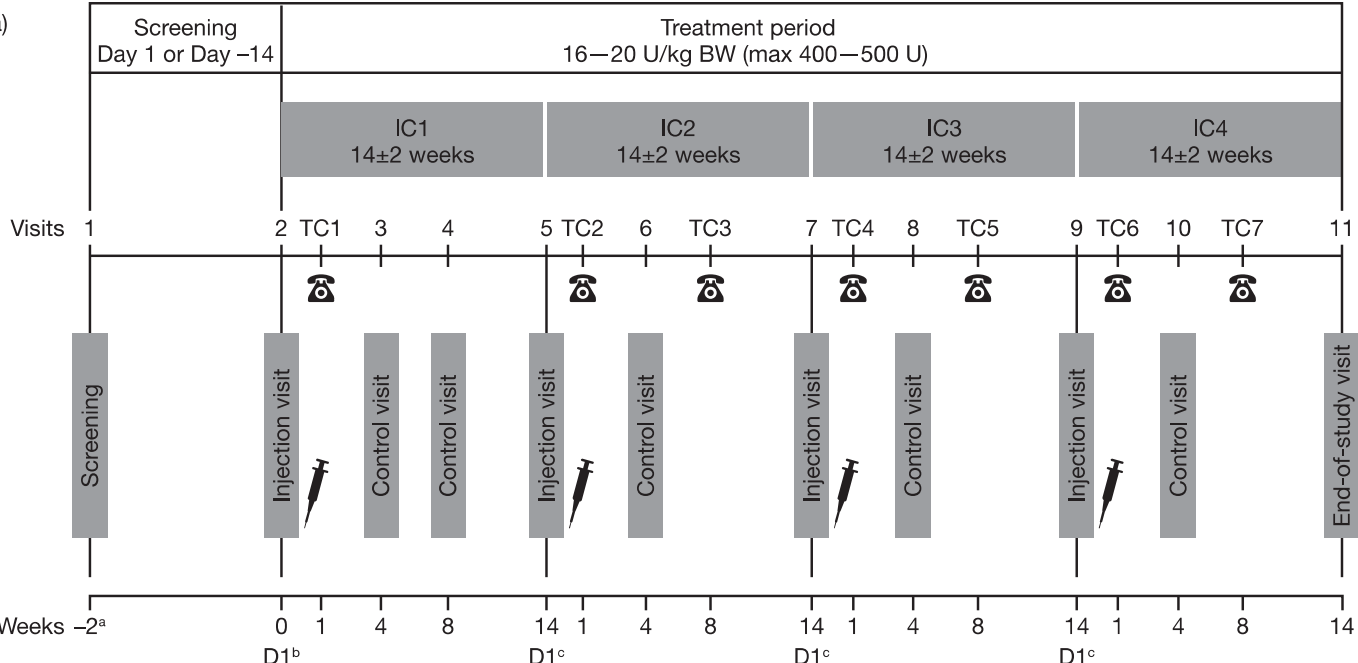

(b)

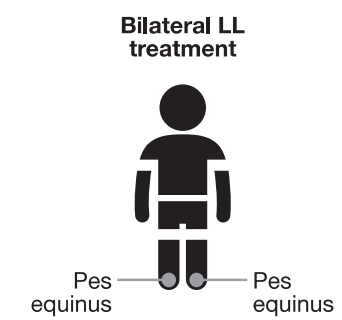

LL treatment only, GMFCS-E\&R I-V (patients continuing from TIM and newly recruited patients)
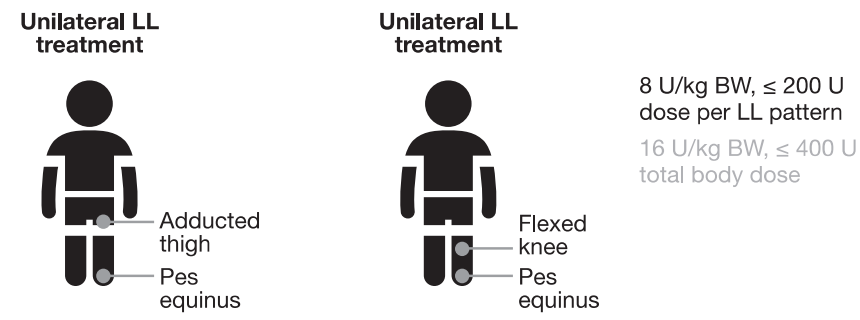

LL and UL treatment, GMFCS-E\&R I-III (newly recruited patients only)
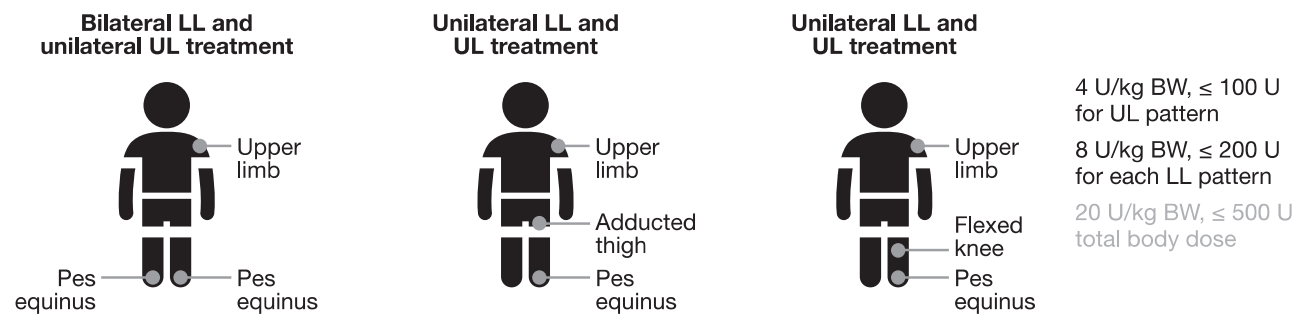

LL and UL treatment, GMFCS-E\&R IV-V (newly recruited patients only)
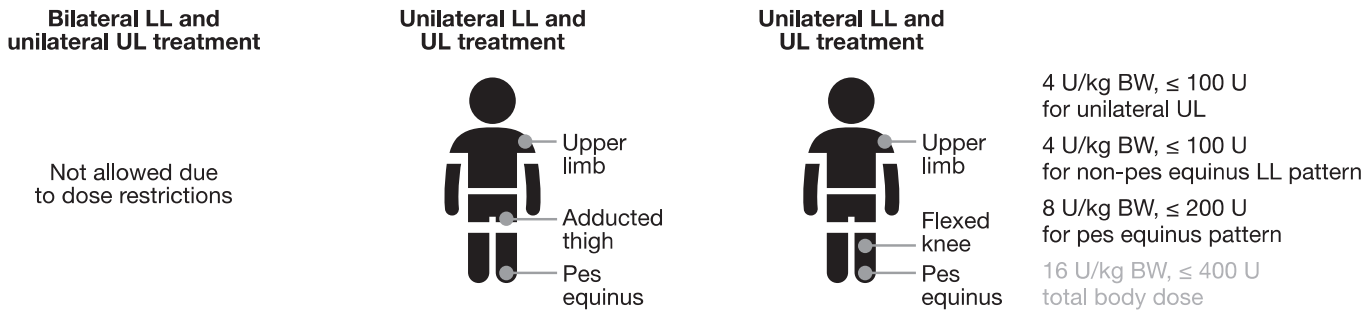

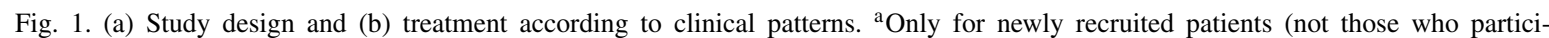
pated in TIM). ${ }^{\mathrm{b}}$ Day 1 of first incobotulinumtoxinA injection. ${ }^{\mathrm{c}}$ Day 1 of the next incobotulinumtoxinA IC. BW = body weight; $D=$ day; GMFCS-E\&R = Gross Motor Function Classification System Expanded and Revised; IC = injection cycle; $\mathrm{kg}=\mathrm{kilogram}$; LL = lower limb; $\max =$ maximum; $\mathrm{TC}=$ telephone contact $\mathrm{TIM}=$ Treatment with IncobotulinumtoxinA in Movement study; $U=$ units; UL = upper limb. 
with standardized incobotulinumtoxinA doses of 12 or $16 \mathrm{U} / \mathrm{kg} \mathrm{BW}$ (maximum 300 or $400 \mathrm{U}$ ) distributed between each pattern depending on ambulatory status. For unilateral UL treatment, investigators were free to distribute the dose as clinically needed within predefined ranges for each clinical pattern (flexed elbow, flexed wrist, clenched fist, thumb-in-palm, and/or pronated forearm), with a total dose of $4 \mathrm{U} / \mathrm{kg}$ BW (maximum $100 \mathrm{U}$ ). UL clinical patterns could be changed at each injection cycle if necessary; however, the same UL was treated consistently throughout the study. The maximum dose of incobotulinumtoxinA per injection site was $25 \mathrm{U}$ for patients with BW $<25 \mathrm{~kg}$ and $50 \mathrm{U}$ for those with BW $\geq 25 \mathrm{~kg}$ (Supplementary Table 1). Incobotulinumtoxin A was supplied as a powder and was reconstituted with sterile saline. The same dilutions were used by each investigator and maintained for all injection cycles. IncobotulinumtoxinA $100 \mathrm{U}$ vials were always reconstituted with $2 \mathrm{~mL}$ saline resulting in incobotulinumtoxinA 5 $\mathrm{U}$ per $0.1 \mathrm{~mL}$ injection solution.

Before the second, third, and fourth injection cycles, the need for reinjection was evaluated by the investigator clinically and confirmed with the parent(s) and patient (if applicable). If eligible, the patient received reinjection within 12-16 weeks of the previous treatment. If the patient was not eligible during this period (no clinical need for reinjection in target limb/clinical pattern determined, AS score $<2$ in treated clinical pattern, no agreement of investigator and patient/parent/caregiver that reinjection was needed, major protocol violation, adverse event for which reinjection would constitute an unacceptably high risk, reinjection could not be performed), they were discontinued from the study and an end-of-study visit was performed.

As continuation of a comprehensive approach to treat limb spasticity was required over the entire study period, patients could continue to receive stable doses of most existing antispastic medications including central muscle relaxants, other medications that were not expected to interfere with the effect of BoNTA, physiotherapy/occupational therapy, (removable) casting, orthotic management, and other rehabilitation measures that were not explicitly excluded. Local anesthesia and/or analgosedation were allowed for the injection procedures to reduce discomfort and pain. To confirm correct placement of the injection needle in this clinical study, all injections had to be guided by at least one of the following: ultrasound, electrical stimulation and electromyography; ultrasound was the preferred guidance technique.

\subsection{Safety}

Safety endpoints were assessed throughout the study and included the occurrence of treatment-emergent adverse events (TEAEs; adverse events with onset or worsening at or after the first injection of incobotulinumtoxinA), TEAEs of special interest (TEAESIs) potentially indicating distant toxin spread, serious TEAEs (TESAEs) and TEAEs assessed as related to treatment by the study investigators. The investigator's Global Assessment of Tolerability (GAT) was an estimation of tolerability based on a 4-point ordinal scale ( $1=$ very good, $2=$ good, $3=$ moderate, $4=$ poor).

BoNT-A antibody testing was conducted only in patients with $\mathrm{BW} \geq 21 \mathrm{~kg}$ because of the substantial blood volume of $14 \mathrm{~mL}$ required for testing. Testing was performed using a fluorescence immunoassay (FIA) to detect binding antibodies against BoNT-A in human serum [26]. Samples positive by FIA were tested for neutralizing antibodies using the validated and highly sensitive mouse ex vivo hemidiaphragm assay (HDA) (sensitivity $<0.5 \mathrm{mU} / \mathrm{mL}$ ) [27].

\subsection{Efficacy}

Assessments of efficacy were preferably made by the same trained investigator at each study visit, and every attempt was made to ensure this. Changes in severity of spasticity were evaluated using the AS for each treated clinical pattern. The AS is a 5-point scale ranging from 0 (no increase in muscle tone) to 4 (limb rigid in flexion or extension) [28].

The investigator's Global Impression of Change of Plantar Flexor Spasticity Scale (GICS-PF) score was assessed using a 7-point Likert scale, which ranged from -3 (very much worse function) to +3 (very much improved function).

The investigator's, child/adolescent's (where applicable), and parent/caregiver's GICS scores for overall LL or UL spasticity were also assessed on a 7-point Likert scale, which ranged from -3 (very much worse) to +3 (very much improved).

The change in patients' gross motor function and ability over time was assessed using Gross Motor Function Measure (GMFM)-66 scores, with higher scores reflecting better function [29].

\subsection{Statistical analyses}

No formal sample size calculation was provided, because analyses were performed in a descriptive and 
exploratory manner with no confirmatory analysis. It was planned that at least 360 patients would be enrolled: $\leq 260$ who had completed TIM and $\geq 100$ who were newly recruited.

Safety variables were analyzed descriptively in the safety evaluation set (SES; patients who received at least one study treatment), and efficacy was evaluated descriptively in the full analysis set (patients from the SES who had at least a baseline AS plantar flexor score available).

The primary safety endpoint of the study was the occurrence of TEAEs, TEAESIs, and TESAEs overall and per injection cycle. Secondary safety endpoints included the occurrence of TEAEs related to treatment as assessed by the investigator, overall and per injection cycle; the occurrence of TEAEs by worst intensity, overall and per injection cycle; and the investigator's GAT estimated at each injection cycle visit before incobotulinumtoxinA was injected, except at the first injection cycle. The incidence of neutralizing antibodies was determined at screening (for patients who completed TIM, data from the TIM screening period were used) and at the end-of-study visit.

Efficacy outcomes comprised secondary and other endpoints and included the change in AS plantar flexor (AS-PF) scores, other LL (knee flexors and thigh adductors) scores, and UL scores from baseline to week 4 of each subsequent visit; GICS scores and investigator's GICS-PF at week 4 of all injection cycles; and the change in GMFM-66 score from the first injection visit to all subsequent injection visits and to the end-of-study visit. For the subset who continued from TIM, changes in AS scores over six cycles were also evaluated. Observed cases analyses were performed, with no imputation for missing values. Statistical tests were two-sided hypothesis tests in general, with 95\% confidence intervals (CIs) and descriptive $p$-values reported, where appropriate. Changes from baseline in AS-PF scores were analyzed using a one-sample t-test and a Wilcoxon signed rank test (both 2-sided, significance level $\alpha=0.05$ ).

Statistical analyses were performed using the statistical analysis system $\left(\mathrm{SAS}^{\circledR}\right)$ software package, version 9.2 or higher (SAS Institute, Cary, NC, USA).

\subsection{Data availability statement}

Key elements of the study protocols, designs, and statistical analysis plans were deposited in the U.S. National Library of Medicine database (www. clinicaltrials.gov, NCT01905683), and EU Clinical
Trials Register (https://eudract.ema.europa.eu/, 2012 -005055-17). All relevant information is contained within this manuscript.

\section{Results}

A total of 30 sites in 12 countries screened 391 patients. Of these, 370 patients were eligible and treated; 124 had completed TIM and 246 were newly recruited (Fig. 2). All patients who had completed TIM received LL treatment only, per protocol; 142

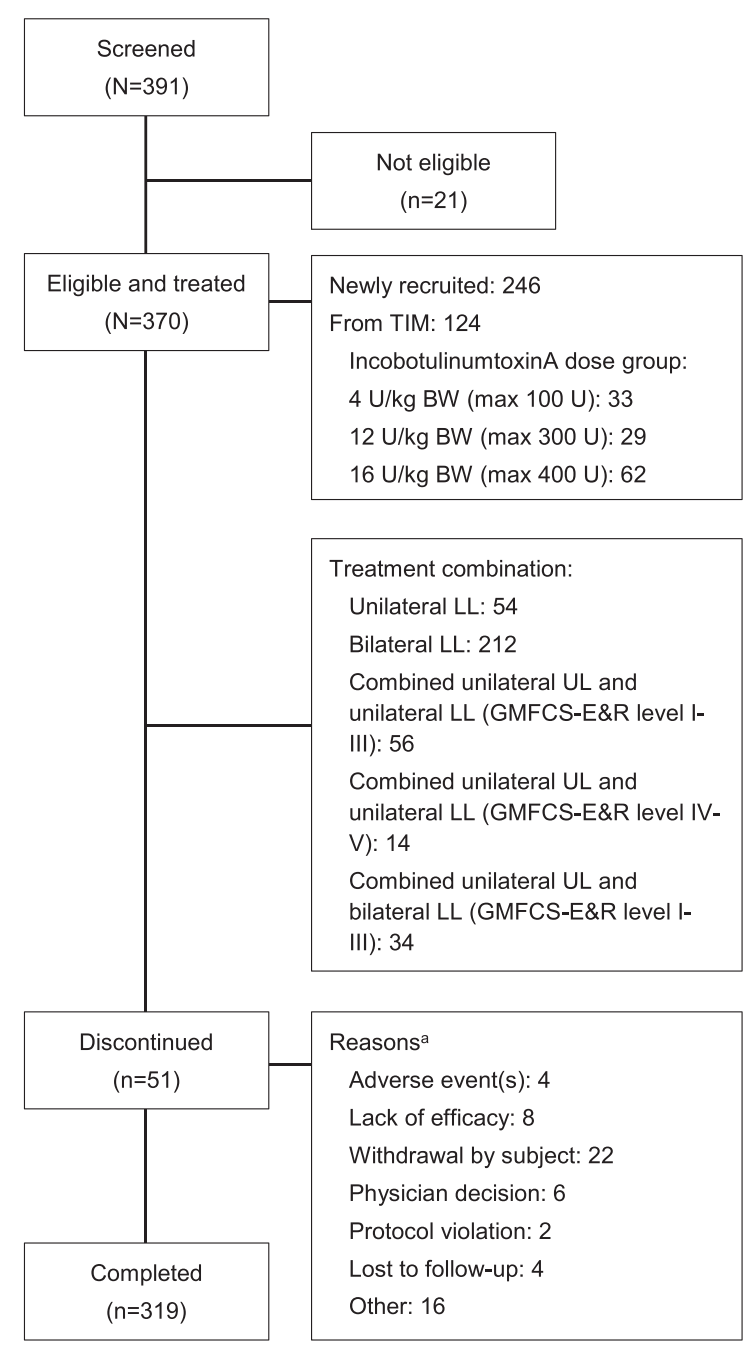

Fig. 2. Patient disposition. ${ }^{a}$ Multiple reasons allowed; discontinuation rates were similar in patients from TIM and newly recruited patients. $\mathrm{BW}=$ body weight; GMFCS-E $\& \mathrm{R}=$ Gross Motor Function Classification System Expanded and Revised; $\mathrm{kg}=$ kilogram; $\mathrm{LL}=$ lower limb; $\max =$ maximum total dose; TIM = Treatment with IncobotulinumtoxinA in Movement study; $U=$ units; $\mathrm{UL}=$ upper limb. 
Table 1

Demographics and baseline characteristics; SES/FAS ${ }^{\mathrm{a}}$

\begin{tabular}{|c|c|c|c|}
\hline Characteristic & $\begin{array}{c}\text { Patients from TIM } \\
(N=124)\end{array}$ & $\begin{array}{l}\text { Newly recruited patients } \\
(N=246)\end{array}$ & $\begin{array}{l}\text { Total population } \\
\quad(N=370)\end{array}$ \\
\hline \multicolumn{4}{|l|}{$\overline{\operatorname{Sex}, n(\%)}$} \\
\hline Male & $68(54.8)$ & $152(61.8)$ & $220(59.5)$ \\
\hline Female & $56(45.2)$ & $94(38.2)$ & $150(40.5)$ \\
\hline Age, years, mean (SD) & $6.9(4.1)$ & $5.8(4.0)$ & $6.2(4.1)$ \\
\hline Weight, kg, mean (SD) & $23.2(12.7)$ & $21.2(13.1)$ & $21.8(13.0)$ \\
\hline Weight $<25 \mathrm{~kg}, \mathrm{n}(\%)$ & $84(67.7)$ & $188(76.4)$ & $272(73.5)$ \\
\hline \multicolumn{4}{|l|}{ GMFCS-E\&R ${ }^{\mathrm{b}}, \mathrm{n}(\%)$} \\
\hline Level I & $25(20.2)$ & $54(22.0)$ & $79(21.4)$ \\
\hline Level II & $35(28.2)$ & $70(28.5)$ & $105(28.4)$ \\
\hline Level III & $29(23.4)$ & $60(24.4)$ & $89(24.1)$ \\
\hline Level IV & $24(19.4)$ & $35(14.2)$ & 59 (15.9) \\
\hline Level V & $11(8.9)$ & $27(11.0)$ & $38(10.3)$ \\
\hline \multicolumn{4}{|l|}{ Affected body side, $n(\%)$} \\
\hline Unilateral right & $11(8.9)$ & $41(16.7)$ & $52(14.1)$ \\
\hline Unilateral left & $13(10.5)$ & $38(15.4)$ & $51(13.8)$ \\
\hline Bilateral & $100(80.6)$ & $167(67.9)$ & $267(72.2)$ \\
\hline Previously received BoNT-A, $n(\%)^{\mathrm{c}}$ & $85(68.5)$ & $119(48.4)$ & $204(55.1)$ \\
\hline \multicolumn{4}{|l|}{ Baseline AS-PF score } \\
\hline Left/right, mean (SD) & $2.7(0.5) / 2.5(0.6)^{\mathrm{d}}$ & $2.5(0.5) / 2.8(0.5)^{\mathrm{d}}$ & $2.8(0.5) / 2.7(0.5)^{\mathrm{d}}$ \\
\hline Left/right, median (interquartile range) & $2(2,3) / 2(2,3)^{\mathrm{d}}$ & $3(3,3) / 3(2,3)^{\mathrm{d}}$ & $3(2,3) / 3(2,3)^{\mathrm{d}}$ \\
\hline \multicolumn{4}{|l|}{ Concomitant diseases } \\
\hline Patients with at least one finding, $n(\%)$ & $90(72.6)$ & $183(74.4)$ & $273(73.8)$ \\
\hline \multicolumn{4}{|l|}{ Most common $^{\mathrm{e}}, n(\%)$} \\
\hline Intellectual disability & $28(22.6)$ & $59(24.0)$ & $87(23.5)$ \\
\hline Epilepsy & $23(18.5)$ & $57(23.2)$ & $80(21.6)$ \\
\hline Strabismus & $23(18.5)$ & $34(13.8)$ & $57(15.4)$ \\
\hline Developmental speech disorder & $11(8.9)$ & $34(13.8)$ & $45(12.2)$ \\
\hline Foot deformity & $12(9.7)$ & $26(10.6)$ & $38(10.3)$ \\
\hline
\end{tabular}

${ }^{\mathrm{a}}$ The SES and the FAS comprise the same patients and are thus interchangeable. ${ }^{\mathrm{b}}$ GMFCS-E\&R level: I, walks without limitations; II, walks with limitations; III, walks using a handheld mobility device; IV, self-mobility with limitations, may use powered mobile; V, transported

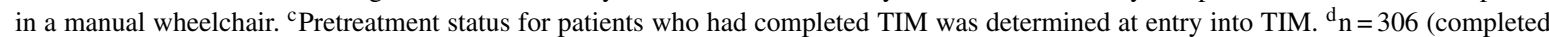
TIM: 110; newly recruited: 196) and 310 (completed TIM: 110; newly recruited: 200) observations for patients treated in left and right plantar flexors, respectively. ${ }^{\mathrm{e}}$ Most common defined as $>10 \%$ in any group. AS-PF=Ashworth Scale of the Plantar Flexors; BoNT-A, botulinum neurotoxin type A; FAS = full analysis set; GMFCS-E\&R=Gross Motor Function Classification System Expanded and Revised; $\mathrm{SD}=$ standard deviation; SES = safety evaluation set; TIM = Treatment with IncobotulinumtoxinA in Movement study.

newly recruited patients received LL treatment only; and 104 newly recruited patients were treated for combined UL and LL spasticity (56 unilateral UL, unilateral LL, GMFCS-E\&R levels I-III; 14 unilateral UL, unilateral LL, GMFCS-E\&R levels IV-V; and 34 unilateral UL, bilateral LL).

Perinatal asphyxia/hypoxia and prematurity were the most common causes of CP $(45.7 \%$ and $25.4 \%$, respectively), and most patients had bilateral spasticity $(72.2 \%)$. Most patients were aged 2-5 years $(55.9 \%)$ and had a mean $\mathrm{BW}<25 \mathrm{~kg}(73.5 \%)$. Demographics and characteristics were generally similar in patients who completed TIM (irrespective of incobotulinumtoxinA dose group in TIM) and those who were newly recruited (Table 1); however, baseline AS-PF scores were slightly lower in patients who completed TIM than in those who were newly recruited, possibly because they had already received two injection cycles of incobotulinumtoxinA in the previous $24-36$ weeks. A total of $55.1 \%$ of patients had previously received BoNT-A treatment. Mean (standard deviation [SD]) total doses of incobotulinumtoxinA were similar for each injection cycle and for patients who completed TIM and those who were newly recruited. Mean doses for the clinical patterns pes equinus, adducted thigh, and flexed knee were slightly greater for patients who completed TIM than for newly recruited patients, a finding explained by the dosing limitations for newly recruited patients who were GMFCS-E\&R levels IV-V. Doses of incobotulinumtoxinA were similar, irrespective of the body side treated. The mean (SD) total observation period was 54.6 (12.6) weeks (range 4-95 weeks) in the total population (52.3 [14.5] weeks in patients who completed TIM and 55.7 [11.4] weeks in newly recruited patients). 


\subsection{Safety}

Across all four injection cycles, 109 patients (29.5\%) experienced a TEAE, ten of whom $(2.7 \%)$ experienced a treatment-related TEAE (Table 2). The proportions of patients experiencing TEAEs were similar among patients who completed TIM $(31.5 \%)$ and new recruits $(28.5 \%)$. The most common TEAEs were nasopharyngitis/pharyngitis, bronchitis, viral respiratory tract infection, and upper respiratory tract infection (Table 3). TEAEs were mild or moderate in intensity in $>95 \%$ of patients. Only one severe TEAE (also a TESAE) was considered to be treatmentrelated by the investigator: one patient with a history of epilepsy had seizures that led to discontinuation; these resolved. There were no major differences across the injection cycles with regard to the pattern of TEAEs, including those that were considered treatment-related.

The incidence of TESAEs was low: $4.3 \%$ across all injection cycles. Two TESAEs in one patient were considered treatment-related (both seizures; one event was also a severe TEAE - see previous paragraph). A total of four TEAESIs were reported for

Table 2

Summary of treatment-emergent adverse events by incobotulinumtoxinA injection cycle; SES

\begin{tabular}{|c|c|c|c|c|c|}
\hline Number of patients, $\mathrm{n}(\%)$ & $\begin{array}{c}\mathrm{IC} 1 \\
N=370\end{array}$ & $\begin{array}{c}\text { IC2 } \\
N=350\end{array}$ & $\begin{array}{c}\text { IC3 } \\
N=340\end{array}$ & $\begin{array}{c}\mathrm{IC} 4 \\
N=323\end{array}$ & $\begin{array}{c}\text { Total }^{\mathrm{a}} \\
N=370\end{array}$ \\
\hline Any TEAE & $53(14.3)$ & $44(12.6)$ & $26(7.6)$ & $31(9.6)$ & $109(29.5)$ \\
\hline Mild & $31(8.4)$ & $25(7.1)$ & $15(4.4)$ & $19(5.9)$ & $57(15.4)$ \\
\hline Moderate & $19(5.1)$ & $17(4.9)$ & $11(3.2)$ & $12(3.7)$ & $48(13.0)$ \\
\hline Severe & $3(0.8)$ & $2(0.6)$ & $0(0.0)$ & $0(0.0)$ & $4(1.1)$ \\
\hline Treatment related & $6(1.6)$ & $5(1.4)$ & $2(0.6)$ & $1(0.3)$ & $10(2.7)$ \\
\hline Any TEAESI & $1(0.3)$ & $2(0.6)$ & $1(0.3)$ & $0(0.0)$ & $3(0.8)$ \\
\hline Treatment related & $1(0.3)$ & $1(0.3)$ & $1(0.3)$ & $0(0.0)$ & $2(0.5)$ \\
\hline Any TESAE & $6(1.6)$ & $5(1.4)$ & $5(1.5)$ & $1(0.3)$ & $16(4.3)$ \\
\hline Treatment related & $0(0.0)$ & $1(0.3)$ & $0(0.0)$ & $0(0.0)$ & $1(0.3)$ \\
\hline $\begin{array}{l}\text { Any TEAE leading to } \\
\text { discontinuation }^{\mathrm{b}}\end{array}$ & $2(0.5)$ & $1(0.3)$ & $1(0.3)$ & $0(0.0)$ & $4(1.1)$ \\
\hline Treatment related & $0(0.0)$ & $1(0.3)$ & $0(0.0)$ & $0(0.0)$ & $1(0.3)$ \\
\hline Any fatal TEAE & $0(0.0)$ & $0(0.0)$ & $0(0.0)$ & $0(0.0)$ & $0(0.0)$ \\
\hline
\end{tabular}

${ }^{\mathrm{a}}$ Total relates to number of patients with TEAEs in any injection cycle. ${ }^{\mathrm{b}}$ TEAEs leading to discontinuation in IC1: moderate upper respiratory tract infection (unrelated) in one patient who completed TIM and tenotomy (unrelated) in one newly recruited patient; IC2: severe seizure (related) in one newly recruited patient; IC3: sinusitis (unrelated) in one newly recruited patient. IC = injection cycle; SES = safety evaluation set; TEAE = treatment-emergent adverse event; TEAESI = TEAE of special interest; TESAE = treatment-emergent serious adverse event; TIM = Treatment with IncobotulinumtoxinA in Movement study.

Table 3

Summary of the most common treatment-emergent adverse events ( $>2 \%$ of total population) after incobotulinumtoxinA injection by MedDRA SOC and preferred term; SES

\begin{tabular}{lccc}
\hline Number of patients, $n(\%)$ & $\begin{array}{c}\text { Patients } \\
\text { from TIM } \\
(N=124)\end{array}$ & $\begin{array}{c}\text { Newly recruited } \\
\text { patients } \\
(N=246)\end{array}$ & $\begin{array}{c}\text { Total } \\
\text { population } \\
(N=370)\end{array}$ \\
\hline Infections and infestations & $30(24.2)$ & $49(19.9)$ & $79(21.4)$ \\
$\quad$ Nasopharyngitis & $10(8.1)$ & $11(4.5)$ & $10(4.1)$ \\
Bronchitis & $4(3.2)$ & $6(2.4)$ & $14(3.7)$ \\
Respiratory tract infection, viral & $3(2.4)$ & $5(2.0)$ & $9(2.4)$ \\
Upper respiratory tract infection & $4(3.2)$ & $5(2.0)$ & $9(2.4)$ \\
Pharyngitis & $3(2.4)$ & $4(1.6)$ & $8(2.2)$ \\
$\quad$ Tonsillitis & $4(3.2)$ & $8(3.3)$ & $8(2.2)$ \\
Gastrointestinal disorders & $4(3.2)$ & $9(3.7)$ & $12(3.2)$ \\
Nervous system disorders & $2(1.6)$ & $6(2.4)$ & $11(3.0)$ \\
General disorders and administration site conditions & $4(3.2)$ & $4(1.6)$ & $10(2.7)$ \\
$\quad$ Pyrexia & $4(3.2)$ & $8(3.3)$ & $8(2.2)$ \\
Skin and subcutaneous tissue disorders & $2(1.6)$ & $6(2.4)$ & $10(2.7)$ \\
Musculoskeletal and connective tissue disorders & $3(2.4)$ & $6(2.4)$ & $9(2.4)$ \\
Respiratory, thoracic, and mediastinal disorders & $3(2.4)$ & $3(1.2)$ & $9(2.4)$ \\
Investigations & $5(4.0)$ & $8(2.2)$ \\
\hline
\end{tabular}

MedDRA = Medical Dictionary for Regulatory Activities; PT = preferred term; SES = safety evaluation set; SOC = system organ class; TIM = Treatment with IncobotulinumtoxinA in Movement study. 
three patients $(0.8 \%$; constipation, three events in two patients; muscular weakness in one patient), all of which were mild or moderate in intensity. Two instances of constipation in one patient and one instance of muscle weakness in one patient were assessed by the investigator as treatment-related. In general, the incidences of TEAEs, TESAEs, TEAESIs, and treatment-related TEAEs were stable across injection cycles and did not increase with repeated cycles (Table 2).

The occurrence of TEAEs leading to discontinuation was very low (four patients, $1.1 \%$ ) and, with the exception of the one instance (a seizure that was severe, serious, and treatment-related, as assessed by the investigator), were nonserious, moderate in intensity, and not related to incobotulinumtoxinA and had resolved by the end-of-study visit. There were no deaths during the study.
Investigator's GAT was "good" or "very good" for $92.0 \%$ of patients at the time of the second injection cycle, $91.8 \%$ at the time of the third injection cycle, and $92.0 \%$ at the time of the fourth injection cycle. Poor tolerability was reported at these times for $1.4 \%$, $1.2 \%$, and $2.8 \%$ of patients, respectively. At the endof-study visit, the GAT was "good" or "very good" for $85.7 \%$ and "poor" for $2.2 \%$ of patients.

\subsection{Antibodies to BoNT-A}

Of the 120 patients with antibody tests, two (one new recruit and one who tested positive at screening in TIM) were positive for neutralizing antibodies at screening, and both had previously received BoNT-A treatments. Four patients were positive for neutralizing antibodies at the end-of-study visit; all had
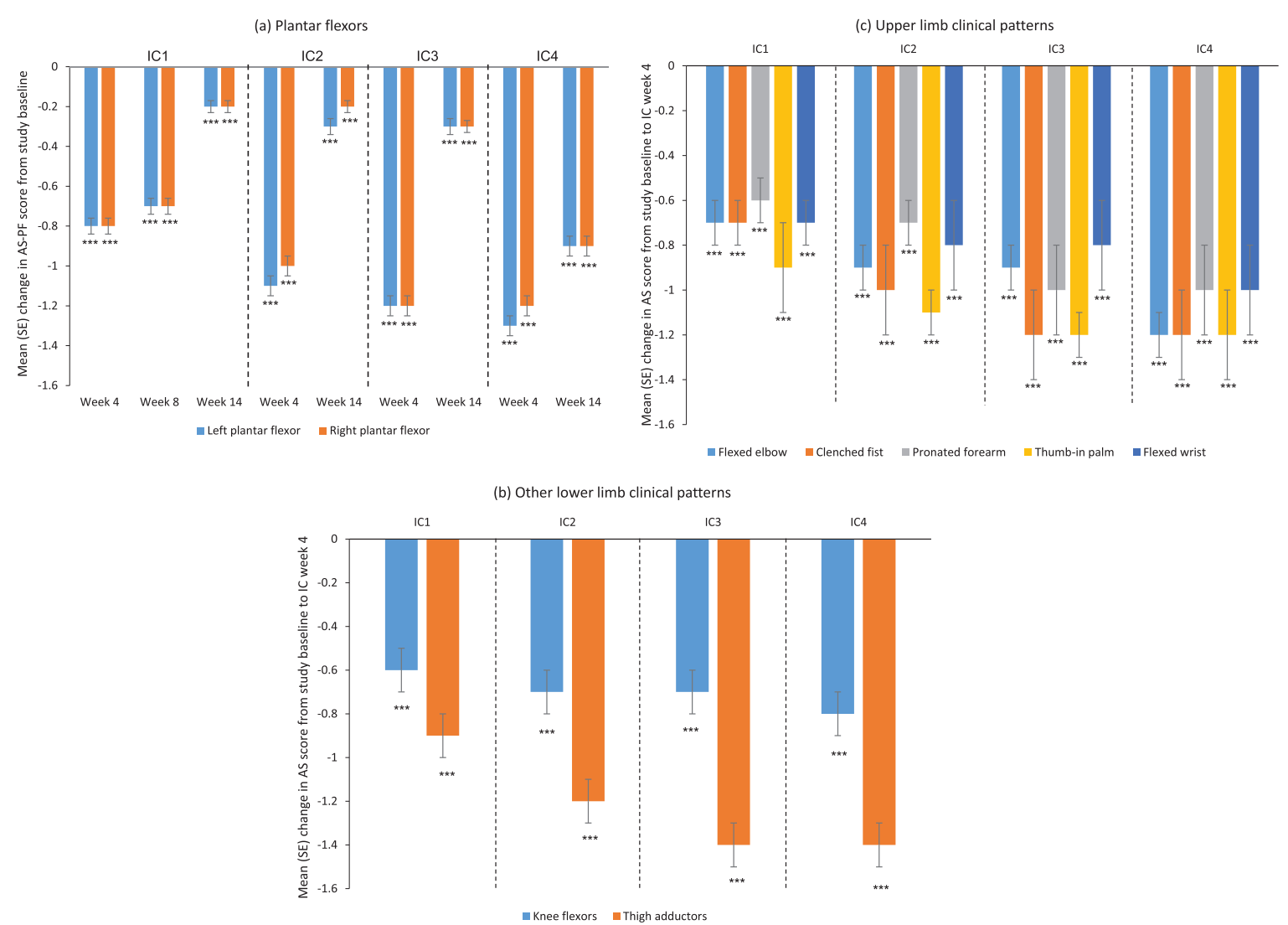

Fig. 3. Change from baseline in AS scores over four incobotulinumtoxinA injection cycles for (a) the plantar flexors, (b) other lower limb clinical patterns, and (c) upper limb clinical patterns; FAS, OC. AS score: 5-point scale from 0 (no increase in muscle tone) to 4 (limb rigid in flexion or extension); a decrease in score indicates improvement. ${ }^{* * *} p<0.001$ versus study baseline, one-sample t-test. AS $=$ Ashworth Scale; AS-PF=Ashworth Scale of the plantar flexors; FAS = full analysis set; $\mathrm{IC}=$ injection cycle; $\mathrm{OC}=$ observed cases; $\mathrm{SE}=$ standard error. 
previously received BoNT-A and two had completed TIM.

No BoNT-A-naïve patients developed neutralizing antibodies after incobotulinumtoxinA treatment, and no patients demonstrated secondary lack of treatment response due to neutralizing antibodies.

\subsection{Efficacy}

Mean AS-PF (left and right) scores improved significantly from baseline to all post-baseline visits $(p<0.001$ for all) and from the day of each injection to all visits of the respective cycle ( $p<0.05$ for all but the week-16 visit of the third injection cycle), particularly the week-4 visit of each cycle (Fig. 3a). A clinically meaningful and consistent treatment response was observed in all four injection cycles, with continuous improvements seen in each cycle versus the previous cycle. Spasticity improvements were also similar for other treated LL and UL clinical patterns (Fig 3b,c). Subgroup analysis according to baseline GMFCSE\&R level was performed for the AS-PF (left and right) and AS elbow flexors. Results indicated that these AS scores improved from baseline in patients of all GMFCS-E\&R levels (data not shown).

When the subgroup of patients who had completed TIM and had therefore received six incobotulinumtoxinA injection cycles was considered, mean AS scores (both the primary and the nonprimary side) were shown to have improved from the baseline of TIM to all visits of the present study $(p<0.001)$, including the baseline visit, and showed consistent spasticity improvements with each injection in all evaluated clinical patterns (Fig. 4).

The GICS-PF change from baseline and investigator's, child's/adolescent's, and parent's/caregiver's GICS ratings all supported that left and right plantar flexor spasticity and LL and UL spasticity, were improved at the 4-week visit after each of the four incobotulinumtoxinA injection cycles (Fig. 5). Consistent with these findings, improvement in plantar flexor spasticity (sum of "minimally improved," "much improved," and "very much improved") according to investigator's GICS-PF was recorded for $>90 \%$ of patients at the week-4 visit of each injection cycle. Good concordance was observed between the investigator's, parent's/caregiver's, and child's/adolescent's GICS, although only about onethird of patients self-reported.

GMFM-66 scores continuously improved from baseline with each injection cycle, with an $8.9 \%$ improvement in motor function seen at the final visit.
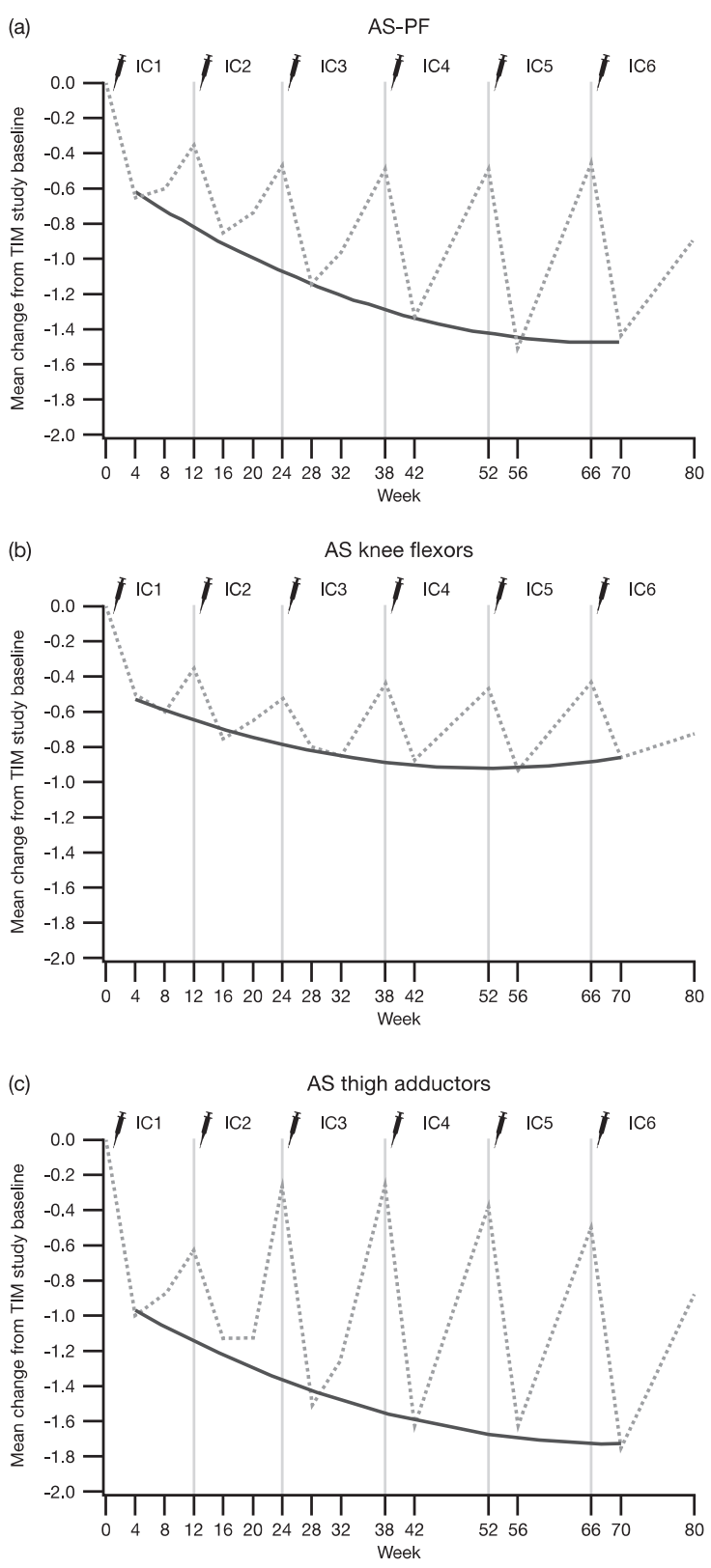

Fig. 4. Change in AS scores of the (a) plantar flexors, (b) knee flexors, and (c) thigh adductors from the TIM study baseline to 4 weeks post-each injection cycle in patients who were treated in TIM and continued into TIMO for a total of six incobotulinumtoxinA injection cycles; FAS, OC. Dashed lines represent the mean change in AS score from the TIM study baseline at each visit, and the solid lines represent the quadratic regression of AS score changes from the TIM study baseline to week 4 in each injection cycle on scheduled time in weeks. AS = Ashworth Scale; AS-PF = Ashworth Scale of the plantar flexors; $\mathrm{CHG}=$ change; FAS $=$ full analysis set; $\mathrm{OC}=$ observed cases; TIM = Treatment with IncobotulinumtoxinA in Movement study; TIMO, Treatment with IncobotulinumtoxinA in Movement Open-Label study. 

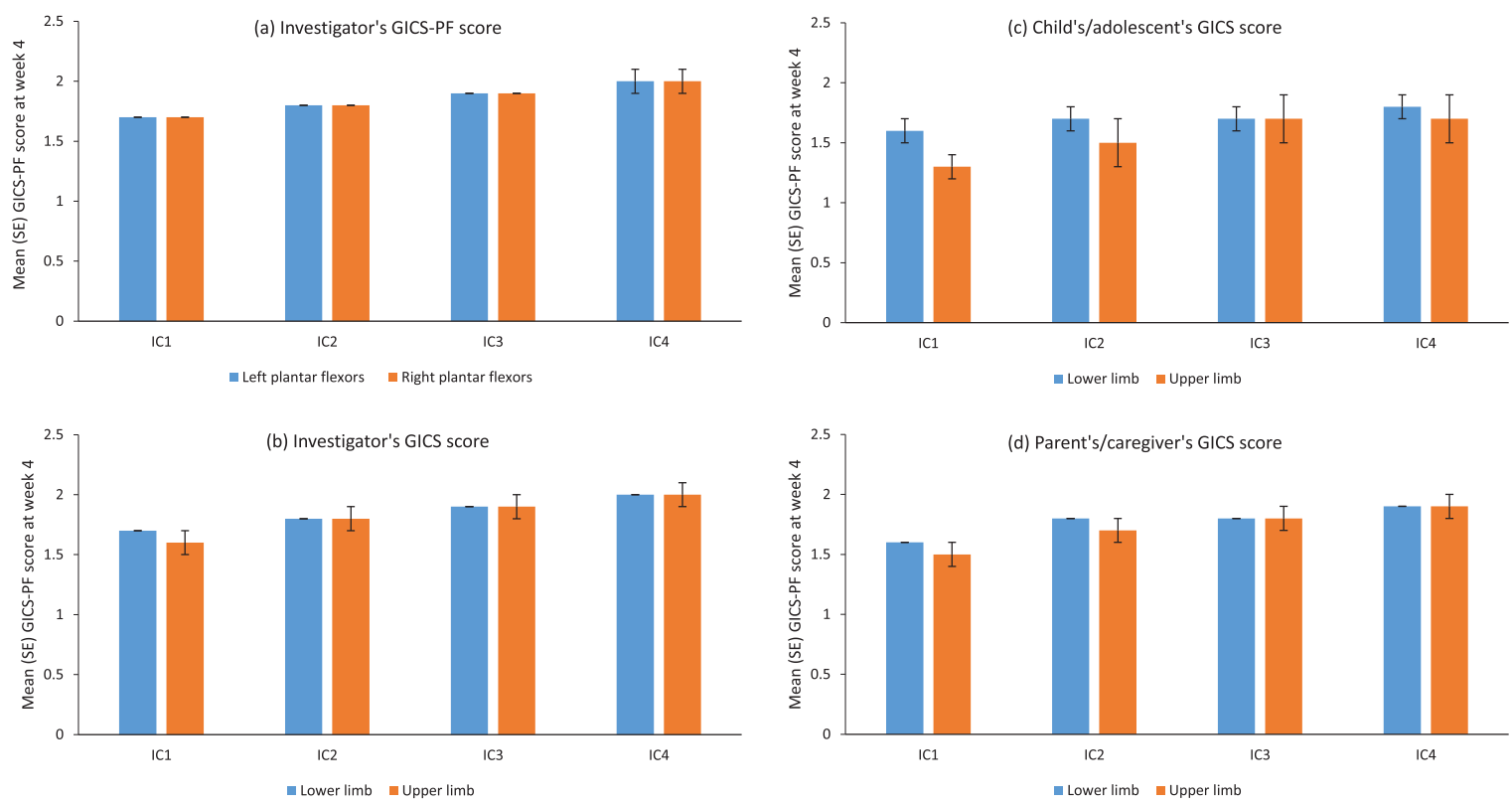

Fig. 5. Global Impression of Change scores for (a) investigator's GICS-PF score at week 4, and GICS score at week 4 as rated by (b) investigators, (c) children/adolescents and (d) parents/caregivers over four incobotulinumtoxinA injection cycles; FAS, OC. GICS/GICS-PF score: -3 , very much worse; -2 , much worse; -1 , minimally worse; 0 , no change; +1 , minimally improved; +2 , much improved; +3 , very much improved. Respondents were asked to rate their overall impression of change in spasticity compared with the condition before the last injection. GICS-PF and GICS results were analysed descriptively with no testing for significance. FAS = full analysis set; GICS = Global Impression of Change Scale; GICS-PF = Global Impression of Change in the plantar flexors; IC = injection cycle; OC = observed cases; $\mathrm{SE}=$ standard error.

Mean (SD) GMFM-66 scores had improved from a baseline of 53.9 (18.9) by 1.5 (3.2), 2.6 (4.0), and 3.8 (5.1) at the start of injection cycles two, three, and four, respectively, and by 4.8 (5.9) at the end-of-study visit.

\section{Discussion}

Results showed that incobotulinumtoxinA was safe and well-tolerated for LL and combined LL plus UL multipattern treatment over four injection cycles (mean [SD] 54.6 [12.6] weeks; range 4-95 weeks) with total body doses up to 16-20 U/kg BW (maximum 400-500 U) in a population of nonambulant (GMFCS levels IV-V, $16 \mathrm{U} / \mathrm{kg} \mathrm{BW}$ ) and ambulant (GMFCS levels I-III, up to $20 \mathrm{U} / \mathrm{kg} \mathrm{BW}$ ) pediatric/adolescent patients with spasticity due to CP. Notably, about one-third of these patients, all of whom were treated with doses up to $16 \mathrm{U} / \mathrm{kg} \mathrm{BW}$ (maximum $400 \mathrm{U}$ ) for LL spasticity, received six cycles of incobotulinumtoxinA. No new or unexpected safety concerns were identified, and the incidence of TEAEs did not increase with repeated treatment and was similar in patients who had com- pleted TIM and in those who were newly recruited.

The TIMO study was designed to investigate the safety and efficacy of incobotulinumtoxinA in a wide range of pediatric patients with CP-related spasticity across all GMFCS levels and affecting a range of clinical patterns in both ULs and LLs, including combined UL and LL patterns, reflecting the real-world distribution of this disorder. It is reassuring that incobotulinumtoxinA treatment was safe and well-tolerated during each of the four to six injection cycles, as evidenced by the $86 \%$ completion rate, in this wide range of young patients, many of whom had ULs and LLs treated simultaneously over long periods of time. Improvements were seen for patients at all disease severities, representing the spectrum of patients presenting in clinical practice. These findings further confirm the safety and good tolerability profile of BoNT-A in children with CP and UL or LL spasticity seen in predominantly shorter-term studies and as recognized by neurology and physiotherapy societies and clinical experts, although generalized weakness can occur [9-11, 20-22, 30-33]. Local injection site discomfort and pain are common but can be managed with local anesthesia and/or analgosedation, as in the current study. The phase 3 
XARA trial also showed that four injection cycles of incobotulinumtoxinA 16-20 U/kg BW (maximum 400-500 U depending on GMFCS level) were safe and well-tolerated in 350 children/adolescents with $\mathrm{CP}$ and uni- or bilateral UL spasticity with or without uni- or bilateral LL spasticity (GMFCS levels I-V) [25].

TEAESIs, potentially indicating distant toxin spread, occurred with low frequency (four TEAESIs were reported in three patients [0.8\%]; three TEAESIs were considered treatment-related) and were mild or moderate in intensity. This finding provides reassurance to those administering and receiving incobotulinumtoxinA treatment and further supports the good tolerability of the drug over multiple injection cycles.

Evaluation of the longer-term safety of incobotulinumtoxinA is also important because BoNT-As are biologic proteins and may induce neutralizing antibodies [23] that have been linked to secondary nonresponse to BoNT-A treatment [34]. Each of the available BoNT-A products has a different composition that may affect its likelihood of inducing neutralizing antibodies [23]. IncobotulinumtoxinA differs from onabotulinumtoxinA and abobotulinumtoxin $\mathrm{A}$ in that it is free from complexing proteins, consisting only of the $150 \mathrm{kDa}$ neurotoxin responsible for its therapeutic effect. In the current study, all four patients who tested positive for neutralizing antibodies according to HDA had a history of previous treatment with BoNT-As other than incobotulinumtoxinA. Neutralizing antibody formation was not observed following up to six cycles of incobotulinumtoxinA (maximum total dose 100-400 U for two cycles [24] then total body doses of up to 400-500 $\mathrm{U}$ for four cycles or total body doses of up to 400-500 $\mathrm{U}$ for four cycles) treatment in any patient who entered TIM or TIMO naïve to BoNT-A. No evidence was found to suggest that patients exposed to incobotulinumtoxinA developed secondary nonresponse to incobotulinumtoxinA due to neutralizing antibodies, which is of importance for the anticipated long-term treatment of spasticity as a life-long condition occurring in young patients with $\mathrm{CP}$.

Results of this study confirm the usual pattern of efficacy seen with BoNT-A [9, 10], with consistent maximal improvement from baseline AS scores 4 weeks after each injection in each of the four injection cycles. These beneficial effects of incobotulinumtoxinA treatment were maintained and enhanced with repeated injection cycles and were observed across patients of all GMFCS-E\&R levels, including those who were nonambulant. This was observed for children/adolescents treated for the LL clinical patterns as well for children who also received UL treatment. Decreased muscle tone was consistently accompanied by improvements in GICS scores as assessed by patients (of whom only about one-third self-reported, since a large number were too young or had insufficient ability to do so), parents/caregivers, and physicians. Similar findings were reported in the XARA trial, in which 350 children/adolescents with $\mathrm{CP}$ and uni- or bilateral UL spasticity with or without uni- or bilateral LL spasticity (GMFCS levels I-V) experienced significant improvements in AS scores from baseline during each of four injection cycles of incobotulinumtoxinA [25].

Changes in motor function, assessed by GMFM66 over time, were consistent with those of previous studies of BoNT-A for LL spasticity, particularly after cycles two to four [35-37]. These results indicate that the functional benefit of incobotulinumtoxinA treatment was maintained through the end of each injection cycle, with no negative impact on gross motor function. GMFM-66 improvements should be considered in the context of the patient's global rehabilitation plan and cannot be solely attributed to incobotulinumtoxinA treatment since many patients also received physiotherapy and rehabilitation therapy. However, improvements were consistent with previously reported minimum clinically important differences of $0.7-1.7$ and 1.2-2.7 for medium and large effects, respectively, in ambulatory children with CP [38]. In children/adolescents with a wider range of $\mathrm{CP}$-related spasticity, including those who were nonambulatory (GMFCS levels I-V, of whom $45 \%$ were GMFCS levels IV-V; $89 \%$ aged $\leq 6$ years), a clinically meaningful change in the GMFM-66 was set at 1.58 , and great improvement was set at 3.71 [39], both within the range of improvements reported at the end of each injection cycle in the current study.

The TIMO study has a number of strengths, including the phase 3 study design that specified standardized yet individualized incobotulinumtoxinA treatment that took into consideration the clinical needs of patients in terms of disease severity and the limbs affected by spasticity. In addition, TIMO enrolled a heterogeneous population of pediatric patients with $\mathrm{CP}$; the proportions of patients at each GMFCS level (I-V) were generally representative of those found in real-world studies [40-42], and patients presented with a range of clinical patterns and limbs requiring treatment for spasticity. Thus, patients were treated according to clinical need and 
received individualized, multipattern treatment that reflected actual clinical practice as much as possible within the constraints of a phase 3 trial. Potential limitations of the TIMO study include the focus on pes equinus spasticity, even though further patterns were allowed; however, this design complemented that of the TIM study, which focused on demonstrating efficacy for LL spasticity. Other potential limitations are that neither gait in ambulatory patients nor quality of life was evaluated.

\section{Conclusions}

IncobotulinumtoxinA at total doses of 16 or $20 \mathrm{U} / \mathrm{kg} \mathrm{BW}$ (maximum 400 or $500 \mathrm{U}$, respectively) were well-tolerated and effective for the multipattern treatment of LL and combined LL plus UL spasticity in nonambulant (GMFCS levels IV-V, $16 \mathrm{U} / \mathrm{kg} \mathrm{BW}$ ) and ambulant (GMFCS levels I-III, up to $20 \mathrm{U} / \mathrm{kg}$ BW) children/adolescents (aged 2-17 years) with CP. No new or unexpected safety concerns were identified over four (up to 64 weeks) or six injection cycles (up to 96 weeks in the subset from TIM). The good safety and tolerability profile, in combination with evidence of efficacy over a wide spectrum of clinical presentations of CP-related spasticity, suggests that incobotulinumtoxinA, when used as adjunct to other treatments, will provide pediatric patients with spasticity with an effective treatment option that can be tailored to meet their changing clinical and developmental needs.

\section{Acknowledgments}

The authors wish to thank all study subjects and investigators. They would like to acknowledge Hanna Dersch and Annette Lehn for statistical support and Caroline Spencer and Amy Rothman Schonfeld (Rx Communications, Mold, UK) for medical writing assistance with the preparation of this manuscript, under the direction of the authors, funded by Merz Pharmaceuticals GmbH.

\section{Conflict of interest}

Petr Kaňovský has received speaker's honoraria from Desitin, Ipsen Biopharmaceuticals, Merz Pharmaceuticals, and Medtronic. Florian Heinen has received speaker's honoraria from Allergan plc, Desitin, Ipsen Biopharmaceuticals, Merz
Pharmaceuticals, and Novartis and unrestricted educational grants from Allergan and Merz Pharmaceuticals. A. Sebastian Schroeder has received speaker's honoraria from and participated in advisory boards for Allergan plc, Ipsen Biopharmaceuticals, and Merz Pharmaceuticals. Henry G. Chambers serves as a consultant for Orthopediatrics Corp and Allergan Corporation. Edward Dabrowski has participated in an advisory board and speaker bureau for Ipsen Biopharmaceuticals. Thorin L. Geister is an employee of Merz Pharmaceuticals GmbH. Angelika Hanschmann is an employee of Merz Pharmaceuticals GmbH. Francisco J. Martinez-Torres is a former employee of Merz North America LLC. Irena Pulte is an employee of Merz Pharmaceuticals $\mathrm{GmbH}$. Marta Banach has served as a consultant and speaker and participated in an advisory board for Merz Pharmaceuticals and has served as a speaker for Allergan, Ipsen, and Kedrion. Deborah Gaebler-Spira has served as a consultant for Teva and Kashiva.

\section{Ethical considerations}

The study protocol, informed consent forms, and other appropriate study-related documents were reviewed and approved by the local independent ethics committees and institutional review boards. Parents/guardians of all patients provided written informed consent, and patients provided assent if applicable.

\section{Supplementary material}

The supplementary material is available in the electronic version of this article: https://dx.doi.org/ 10.3233/PRM-210041.

\section{References}

[1] Sadowska M, Sarecka-Hujar B, Kopyta I. Cerebral palsy: current opinions on definition, epidemiology, risk factors, classification and treatment options. Neuropsychiatr Dis Treat. 2020;16:1505-18. doi: 10.2147/NDT.S235165

[2] Surveillance of Cerebral Palsy in Europe. Surveillance of cerebral palsy in Europe: a collaboration of cerebral palsy surveys and registers. Surveillance of Cerebral Palsy in Europe (SCPE). Dev Med Child Neurol. 2000;42(12):81624. doi: 10.1017/s0012162200001511

[3] Hagglund G, Wagner OP. Development of spasticity with age in a total population of children with cerebral palsy. BMC Musculoskelet Disord. 2008;9:150-9. doi: 10.1186/1471-2474-9-150 
[4] Bar-On L, Molenaers G, Aertbeliën E, Van Campenhout A, Feys H, Nuttin B, et al. Spasticity and its contribution to hypertonia in cerebral palsy. Biomed Res Int. 2015;2015:317047. doi: 10.1155/2015/317047

[5] Lampe R, Mitternacht J. Research on the performance of the spastic calf muscle of young adults with cerebral palsy. J Clin Med Res. 2011;3(1):8-16. doi: 10.4021/ jocmr 483 w

[6] Horsch A, Götze M, Geisbüsch A, Beckmann N, Tsitlakidis $\mathrm{S}$, Berrsche G, et al. Prevalence and classification of equinus foot in bilateral spastic cerebral palsy. World J Pediatr. 2019;15(3):276-80. doi: 10.1007/s12519-019-00238-2

[7] Rethlefsen SA, Blumstein G, Kay RM, Dorey F, Wren TA. Prevalence of specific gait abnormalities in children with cerebral palsy revisited: influence of age, prior surgery, and Gross Motor Function Classification System level. Dev Med Child Neurol. 2017;59(1):79-88. doi: 10.1111/dmen.13205

[8] BMJ Best Practice. Cerebral palsy [cited 2021 Feb 4]. Available from: https://bestpractice.bmj.com/topics/en-us/674.

[9] Fehlings D, Novak I, Berweck S, Hoare B, Stott NS, Russo RN. Cerebral Palsy Institute. Botulinum toxin assessment, intervention and follow-up for paediatric upper limb hypertonicity: international consensus statement. Eur J Neurol. 2010;17(Suppl 2):38-56. doi: 10.1111/j.14681331.2010.03127.x

[10] Love SC, Novak I, Kentish M, Desloovere K, Heinen F, Molenaers G, et al., Cerebral Palsy Institute. Botulinum toxin assessment, intervention and after-care for lower limb spasticity in children with cerebral palsy: international consensus statement. Eur J Neurol. 2010;17(Suppl 2):9-37. doi: 10.1111/j.1468-1331.2010.03126.x

[11] Delgado MR, Hirtz D, Aisen M, Ashwal S, Fehlings DL, McLaughlin J, et al. Practice parameter: pharmacologic treatment of spasticity in children and adolescents with cerebral palsy (an evidence-based review): report of the Quality Standards Subcommittee of the American Academy of Neurology and the Practice Committee of the Child Neurology Society. Neurology. 2010;74(4):336-43. doi: 10.1212/WNL.0b013e3181cbcd2f

[12] Heinen F, Desloovere K, Schroeder AS, Berweck S, Borggraefe I, van Campenhout A, et al. The updated European Consensus 2009 on the use of Botulinum toxin for children with cerebral palsy. Eur J Paediatr Neurol. 2010; 14(1):45-66. doi: 10.1016/j.ejpn.2009.09.005

[13] National Collaborating Centre for Women's and Children's Health. Commissioned by the National Institute for Health and Clinical Excellence. Spasticity in children and young people with non-progressive brain disorders: management of spasticity and co-existing motor disorders and their early musculoskeletal complications. NICE clinical guideline. July 2012 [cited $2021 \mathrm{Feb} 11$ ]. Available from: https://www.nice.org.uk/guidance/cg145/evidence/fullguideline-updated-november-2016-pdf-186774301.

[14] Botox ${ }^{\circledR} 100$ U. Summary of product characteristics. Bucks: Allergan Ltd, 2020 [cited $2021 \mathrm{Feb}$ 4]. Available from: https://www.medicines.org.uk/EMC/medicine/112/SPC/.

[15] BOTOX (onabotulinumtoxinA) for injection, for intramuscular, intradetrusor, or intradermal use. Highlights of prescribing information - Botox ${ }^{\circledR}$. Dublin: Allergan Inc., 2019 [cited 2021 Feb 23]. Available from: https://media.allergan.com/actavis/actavis/media/allerganpdf-documents/product-prescribing/20190620-BOTOX100-and-200-Units-v3-0USPI1145-v2-0MG1145.pdf

[16] Dysport ${ }^{\circledR}$ 500U. Summary of product characteristics. Cambridge, MA: Ipsen Biopharm Ltd, 2020 [cited
2021 Jan 19]. Available from: https://www.medicines. org.uk/emc/medicine/32114.

[17] DYSPORT (abobotulinumtoxinA) for injection, for intramuscular use. Highlights of prescribing information. Cambridge, MA: Ipsen Biopharm Ltd, 2020 [cited 2021 Feb 4]. Available from: https://www.ipsen.com/websites/ Ipsen_Online/wp-content/uploads/2020/07/10002305/DYSUS-004998_Dysport-PI-July-2020.pdf

[18] XEOMIN (incobotulinumtoxinA) for injection, for intramuscular or intraglandular use: US prescribing information. Raleigh, NC: Merz Pharmaceuticals LLC, 2020 [cited 2021 Feb 5]. Available from: https://www.accessdata. fda.gov/drugsatfda_docs/label/2020/125360s078lbl.pdf

[19] Merz Pharma UK Ltd. Xeomin 200 units powder for solution for injection. Herts: Merz Pharma UK Ltd, 2020 [cited 2021 Feb 05]. Available from: https:// www.medicines.org.uk/emc/product/2162/smpc

[20] Delgado MR, Tilton A, Russman B, Benavides O, Bonikowski M, Carranza J, et al. AbobotulinumtoxinA for equinus foot deformity in cerebral palsy: a randomized controlled trial. Pediatrics. 2016;137(2):e20152830. doi: 10.1542/peds.2015-2830

[21] Delgado MR, Tilton A, Carranza-Del Río J, Dursun N, Bonikowski M, Aydin R, et al., Dysport in PUL study group. Efficacy and safety of abobotulinumtoxinA for upper limb spasticity in children with cerebral palsy: a randomized repeat-treatment study. Dev Med Child Neurol. 2021;63(5):592-600. doi: 10.1111/dmcn.14733

[22] Hong BY, Chang HJ, Lee SJ, Lee S, Park JH, Kwon JY. Efficacy of repeated botulinum toxin type A injections for spastic equinus in children with cerebral palsy - a secondary analysis of the randomized clinical trial. Toxins (Basel). 2017;9(8):253. doi: 10.3390/toxins9080253

[23] Frevert J. Pharmaceutical, biological, and clinical properties of botulinum neurotoxin type A products. Drugs R D. 2015;15(1):1-9. doi: 10.1007/s40268-014-0077-1

[24] Heinen F, Kaňovský P, Schroeder AS, Chambers HG, Dabrowski E, Geister TL, et al. IncobotulinumtoxinA for the treatment of lower-limb spasticity in children and adolescents with cerebral palsy: a phase 3 study. J Pediatr Rehabil Med. 2021;14(2):183-97. doi: 10.3233/PRM-210040

[25] Dabrowski E, Chambers HG, Gaebler-Spira D, Banach M, Kaňovský P, Dersch $\mathrm{H}$, et al. Efficacy and safety of incobotulinumtoxinA for upper- or combined upperand lower-limb spasticity in children and adolescents with cerebral palsy: results of the phase 3 XARA study. Toxicon. 2021;190(Suppl 1):S14-S15. doi: 10.1016/ j.toxicon.2020.11.369

[26] Göschel H, Wohlfarth K, Frevert J, Dengler R, Bigalke H. Botulinum A toxin therapy: neutralizing and nonneutralizing antibodies - therapeutic consequences. Exp Neurol. 1997;147(1):96-102. doi: 10.1006/exnr.1997.6580

[27] Sesardic D, Jones RG, Leung T, Alsop T, Tierney R. Detection of antibodies against botulinum toxins. Mov Disord. 2004;19(Suppl 8):S85-91. doi: 10.1002/mds.20021

[28] Ashworth B. Preliminary trial of carisoprodol in multiple sclerosis. Practitioner. 1964;192:540-2.

[29] Russell DJ, Avery LM, Rosenbaum PL, Raina PS, Walter $\mathrm{SD}$, Palisano RJ. Improved scaling of the gross motor function measure for children with cerebral palsy: evidence of reliability and validity. Phys Ther. 2000;80(9):873-85. doi: 10.1093/ptj/80.9.873

[30] Hoare BJ, Wallen MA, Imms C, Villanueva E, Rawicki HB, Carey L. Botulinum toxin $\mathrm{A}$ as an adjunct to treatment in the management of the upper limb in 
children with spastic cerebral palsy (UPDATE). Cochrane Database Syst Rev. 2010;2010(1):CD003469. doi: 10.1002/ 14651858.CD003469.pub4

[31] Delgado MR, Bonikowski M, Carranza J, Dabrowski E, Matthews D, Russman B, et al. Safety and efficacy of repeat open-label abobotulinumtoxinA treatment in pediatric cerebral palsy. J Child Neurol. 2017;32(13):1058-64. doi: $10.1177 / 0883073817729918$

[32] Lukban MB, Rosales RL, Dressler D. Effectiveness of botulinum toxin A for upper and lower limb spasticity in children with cerebral palsy: a summary of evidence. J Neural Transm (Vienna). 2009;116(3):319-31. doi: 10.1007/s00702-008-0175-8

[33] Association of Paediatric Chartered Physiotherapists. Evidence-based guidance for physiotherapists. The use of Botulinum Toxin in Children with Neurological Conditions [cited 2021 Aug 06]. Available from: https:// apcp.csp.org.uk/system/files/use_of_botulinum_toxin.pdf

[34] Mathevon L, Declemy A, Laffont I, Perennou D. Immunogenicity induced by botulinum toxin injections for limb spasticity: A systematic review. Ann Phys Rehabil Med. 2019;62(4):241-51. doi: 10.1016/j.rehab.2019.03.004

[35] Baker R, Jasinski M, Maciag-Tymecka I, MichalowskaMrozek J, Bonikowski M, Carr L, et al. Botulinum toxin treatment of spasticity in diplegic cerebral palsy: a randomized, double-blind, placebo-controlled, dose-ranging study. Dev Med Child Neurol. 2002;44(10):666-75. doi: $10.1017 / \mathrm{s} 0012162201002730$

[36] Chang HJ, Hong BY, Lee SJ, Lee S, Park JH, Kwon JY. Efficacy and safety of letibotulinum toxin A for the treatment of dynamic equinus foot deformity in children with cerebral palsy: a randomized controlled trial. Toxins (Basel). 2017;9(8):252. doi: 10.3390/toxins 9080252
[37] Kim K, Shin HI, Kwon BS, Kim SJ, Jung IY, Bang MS. Neuronox versus BOTOX for spastic equinus gait in children with cerebral palsy: a randomized, doubleblinded, controlled multicentre clinical trial. Dev Med Child Neurol. 2011;53(3):239-44. doi: 10.1111/j.14698749.2010.03830.x

[38] Oeffinger D, Bagley A, Rogers S, Gorton G, Kryscio $\mathrm{R}$, Abel $\mathrm{M}$, et al. Outcome tools used for ambulatory children with cerebral palsy: responsiveness and minimum clinically important differences. Dev Med Child Neurol. 2008;50(12):918-25. doi: 10.1111/j.14698749.2008.03150.x

[39] Wang HY, Yang YH. Evaluating the responsiveness of 2 versions of the gross motor function measure for children with cerebral palsy. Arch Phys Med Rehabil. 2006;87(1):516. doi: 10.1016/j.apmr.2005.08.117

[40] Reid SM, Carlin JB, Reddihough DS. Using the Gross Motor Function Classification System to describe patterns of motor severity in cerebral palsy. Dev Med Child Neurol. 2011;53(11):1007-12. doi: 10.1111/j.14698749.2011.04044.x

[41] Bugler KE, Gaston MS, Robb JE. Distribution and motor ability of children with cerebral palsy in Scotland: a registry analysis. Scott Med J. 2019;64(1):16-21. doi: $10.1177 / 0036933018805897$

[42] Himmelmann K, Beckung E, Hagberg G, Uvebrant P. Gross and fine motor function and accompanying impairments in cerebral palsy. Dev Med Child Neurol. 2006;48(6):417-23. doi: 10.1017/S0012162206000922 